\title{
Global Diversity and Local Consensus in Status Beliefs: The Role of Network Clustering and Resistance to Belief Change
}

\author{
André Grow, ${ }^{a}$ Andreas Flache, ${ }^{b}$ Rafael P. M. Wittek ${ }^{b}$
}

a) KU Leuven; b) University of Groningen

Abstract: Formal models of status construction theory suggest that beliefs about the relative social worth and competence of members of different social groups can emerge from face-to-face interactions in task-focused groups and eventually become consensual in large populations. We propose two extensions of earlier models. First, we incorporate the microlevel behavioral assumption of status construction theory that people can become resistant to belief change when a belief appears consensual in their local social environment. Second, we integrate the insight that the macro-level social structure of face-to-face interactions in large populations often is a clustered network structure. Computational experiments identify an outcome that was not anticipated by earlier formalizations. The combination of network clustering at the macrolevel and resistance to belief change at the microlevel can constrain the diffusion of status beliefs and generate regional variation in status beliefs. Further experiments identify conditions under which this outcome obtains.

Keywords: status construction theory; status beliefs; network clustering; agent-based computational modelling

Citation: Grow, André, Andreas Flache, and Rafael P. M. Wittek. 2017. "Global Diversity and Local Consensus in Status Beliefs: The Role of Network Clustering and Resistance to Belief Change." Sociological Science 4: 611-640.

Received: July 30, 2017

Accepted: September 23, 2017

Published: November 6, 2017

Editor(s): Jesper Sørensen, Delia Baldassarri

DOI: $10.15195 / \mathrm{v} 4 . \mathrm{a} 25$

Copyright: (C) 2017 The Author(s). This open-access article has been published under a Creative Commons Attribution License, which allows unrestricted use, distribution and reproduction, in any form, as long as the original author and source have been credited. (C)(i)

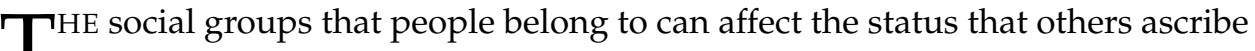
1 to them. In the United States, for example, whites are often more respected and viewed as more competent than blacks (Feagin 1991; Steele and Aronson 1995), whereas in Hungary, members of the Hungarian majority are often more respected and viewed as more competent than members of the Roma minority (Grow, Takács, and Pál 2016; Neményi 2007). Status differences have important implications for social inequality. High-status individuals often fare better in the educational system and have better chances on the labor market (Foschi, Lai, and Sigerson 1994; MossRacusin et al. 2012; Schmader 2002; Steele and Aronson 1995), frequently are in better health, report higher subjective well-being (Anderson et al. 2012; Fournier 2009; Ghaed and Gallo 2007; Huo, Binning, and Molina 2010; Tay and Diener 2011), and tend to have a higher life expectancy (Marmot 2004) than lower-status individuals. Social scientists therefore have a long-standing interest in explaining how social distinctions attain status value.

Traditionally, sociologists have assumed that individuals attain status through the use of valuable resources as a currency in exchange for respect and deference (e.g., Blau 1964; Goode 1979). Applied to the population level, this explanation implies that status differentiation between social groups is the result of systematic resource differences between them. In the case of gender, for example, it has been argued that certain religious beliefs, inheritance practices, and modes of production create social structures in which resources and power become concentrated among 
men, thereby leading to differences in the worth attributed to the different sexes (Hendrix and Hossain 1988; Sanderson, Heckert, and Dubrow 2005; Stover and Hope 1984). Similarly, differences in the modes of production and inheritance customs across societies have been assumed to create variation in the resources and power that the elderly control. This might partly explain why older people have low status in some societies but high status in others (Balkwell and Balswick 1981; Ishii-Kuntz and Lee 1987).

More recent research based on status construction theory (SCT) has described processes that might lead to status differentiation between social groups, even in the absence of any systematic resource and power differences between them (Grow, Flache, and Wittek 2015; Mark, Smith-Lovin, and Ridgeway 2009; Ridgeway 2000; Ridgeway and Correll 2006). SCT focuses on interactions in small groups with a collective task focus (e.g., work teams, student learning groups, and neighborhood organizations) as building blocks of society. It holds that such groups can spontaneously develop hierarchies of influence and deference, in which some individuals are perceived as more respected and more competent than others. When such differentiation occurs consistently between members of different social categories, even if only by accident, individuals can come to believe that the social distinction is generally associated with differences in respect and competence. Once emerged, such beliefs can diffuse throughout the population because people carry them into new interaction contexts, treat new interaction partners accordingly, and thereby create hierarchies that teach their beliefs to others (Ridgeway 2000). Indeed, modelling work by Mark et al. (2009) formalizes this intuition and shows that status beliefs have a strong tendency to emerge and to diffuse widely under minimal assumptions about the microprocess of hierarchy formation and belief diffusion. This result obtains even in the absence of systematic resource differences between the members of different social categories.

Mark et al.'s (2009) formal argument that status construction processes are a potent force in the creation of status differentiation is intriguing and sheds new light on possible sources of social inequality. However, their analysis did not incorporate a central assumption of SCT. Mark et al. (2009) assumed that a single interactional experience with members of a different social category is sufficient for people to acquire a new status belief or to lose an existing belief if a new experience contradicts it. By contrast, SCT holds that people consider multiple experiences and that belief acquisition and maintenance depend on how consensual individuals perceive a given belief in their social environment (Ridgeway 2000; Ridgeway and Correll 2006). That is, although people might adopt a given belief from an experience they make in a local interaction context, they are unlikely to maintain this belief if it is not reinforced in subsequent interactions that make it appear consensual in their social environment. Once a given belief appears consensual, people can then become resistant to changing it, even in the light of occasionally disconfirming evidence. We propose here an extension of Mark et al.'s (2009) model that incorporates these processes of reinforcement in belief acquisition and maintenance. As we will show, this extension gives rise to an intriguing micro-macro interaction. Even under the assumption that locally established status beliefs become resistant to change (from here on also called belief inertia, for short), Mark et al.'s fundamental result that 
status beliefs eventually become globally consensual can be obtained. However, it turns out that this is no longer the case if we also relax a crucial assumption they made about the macrostructural pattern of social relations in a population.

Next to assuming that people only consider a single interactional experience in belief acquisition and loss, Mark et al. (2009) assumed complete and unstructured interaction networks in their actor populations so that interactions between any two members of the population were equally likely at any point in time, regardless of the size of the population. In reality, people tend to interact only with a small subset of a large population, typically with others who are connected within the same local cluster of network ties (e.g., Davis 1970; Faust et al. 1999; Festinger, Schachter, and Back 1950; Granovetter 1973; Watts 1999; Wong, Pattison, and Robins 2006). Related simulation studies have shown that such network clustering can impose powerful constraints on how fast and widely social objects such as information, opinions, cultural traits, and reputations diffuse throughout society (e.g., DellaPosta, Shi, and Macy 2015; Flache and Macy 2011a, 2011b; Parisi, Cecconi, and Natale 2003). This is especially the case if the adoption of such objects requires reinforcement from multiple adopting neighbors (Centola and Macy 2007).

We expect that network clustering will have a similar effect on the diffusion of status beliefs under our extension of the microprocess of status belief diffusion. Network clustering creates dense local interaction structures that can quickly diffuse any incidentally created belief among the members of local communities. This results in a social reality that renders the belief highly consensual within a community. If belief acquisition and maintenance require some consensus in peoples' immediate social environment, the emergent local consensus can, in turn, ward off potential influence from less frequently occurring interactions with members of other communities in which different beliefs might have emerged. As a consequence, in different local regions of the network, different status beliefs emerge and persist without becoming consensual in the wider population. Yet this can only happen if people are resistant to changing beliefs that appear consensual in their social environment. Network clustering in itself is not sufficient to prevent status beliefs to diffuse and become widely accepted in the larger population; it needs to be accompanied by the extension of the microprocess of status belief diffusion we propose here.

Taken together, we suggest that the processes that SCT describes not only lead to the emergence of widespread consensus in status beliefs, as suggested by Mark et al. (2009). They can also lead to the emergence of persistent diversity in status beliefs if network clustering and belief inertia are considered jointly. In the remainder of this article, we extend the model of Mark et al. (2009) to incorporate network clustering as a new macrolevel condition and belief inertia as a new microlevel behavioral assumption. In reality, there can be multiple factors that lead social networks to be clustered. For simplicity, in developing our model, we focus on the constraints that spatial distances impose on interactions between people as one of the most important sources of network clustering in large and geographically distributed populations. As we will argue, these spatially clustered networks exhibit structural features that are found in many empirical social networks. To show the logical consistency of our argument, we submit the new model to computational 
experiments, in which we illustrate how the changes that we propose affect the belief diffusion process separately and jointly. We start with presenting the central premises of SCT, discussing properties of spatially clustered network structures, and arguing in detail how these properties affect the diffusion of status beliefs when they are combined with belief inertia. Subsequently, we describe the model and present the results of our simulation experiments. We close with discussing the implications of our work for future research. In particular, we discuss how our results might change if future research would also consider other sources of network clustering next to spatial distances.

\section{An Informal Theoretical Account}

\section{SCT's Behavioral and Cognitive Assumptions}

SCT is part of the expectation states framework (Berger et al. 1977; Berger, Wagner, and Webster Jr. 2014) that centers on the emergence of status differentiation in groups with a collective task focus. Collective task focus means that group members perceive the successful completion of some important task as the primary purpose of their membership in the group and that success can only be accomplished through teamwork. Within the expectation states framework, SCT describes social processes by which salient social distinctions can become sources of status (i.e., attain status value) that shape hierarchies and interaction patterns in task-focused contexts.

Research in SCT usually focuses on social distinctions that create at least two mutually exclusive categories that are easy to discern during face-to-face interaction (e.g., sex and race). The status value of such distinctions is determined by the distribution of status value beliefs (or simply status beliefs) in a population (Ridgeway 1991). Status beliefs are assumptions that "associate greater social esteem and competence with people in one category than with those from another" (Ridgeway et al. 2009:44). A distinction has attained status value when the widespread belief exists that members of one category are relatively more respected and competent than members of the other category (Ridgeway and Balkwell 1997). Gender, for instance, has status value in populations in which men are widely believed to be more respected and competent than women. SCT argues that interactions between members of different social categories in task-focused groups can spontaneously create, reproduce, and diffuse status beliefs. Three assumptions of SCT are central to this. Our discussion of the first two assumptions is in line with that by Mark et al. (2009). With our third assumption, we build upon but extend the way Mark et al. (2009) model belief acquisition and belief loss and put stronger emphasis on the importance of perceived consensus for belief formation and maintenance.

First, the status hierarchy emergence assumption holds that task-focused groups tend to quickly develop hierarchical differences in respect and influence in their efforts to reach their collective goals (e.g., Bales 1970). The reason is that in such groups, individuals act "as if one of the[ir] subtasks is to decide who has high and who has low ability at the [collective] task-thus to take advantage of high ability members and not to be misled by low ability members" (Driskell Jr. 1982:232). They therefore search for cues that might signal competence differences. If such cues 
exist, the group's hierarchy in terms of respect and influence will quickly reflect assumptions about relative competence (Ridgeway 1991). Competence cues do not always reflect actual competence differences (Webster and Hysom 1998). Confident behavior, for instance, is often assumed to indicate knowledge and competence. Individuals who act in a confident manner are therefore frequently perceived as more competent, even when their contributions are qualitatively not different from contributions made by individuals who act less confident (cf. Carli, LaFleur, and Loeber 1995). Thus, hierarchies can emerge even when there are no objective bases for status differentiation.

Second, the status belief effect assumption holds that in socially differentiated interactions, status beliefs can affect assumptions about respect and competence. For example, when men and women interact in a small group setting and some individuals believe that women are generally more respected and competent than men, they will be more attentive to and accepting of the suggestions of female group members. This makes it likely that hierarchies of respect and influence emerge that reflect the belief that females are more respected and competent than males (for an overview of research on this phenomenon, see Wagner and Berger [2002]).

Third, the perceived consensus and belief change assumption holds that the observation of hierarchical differentiation between particular members of different social categories can induce beliefs about the respectability and competence of members of the categories in general. That is, individuals tend to infer respect and competence from hierarchical differentiation in face-to-face interaction (Ridgeway 2000). When such differentiation coincides with differences in a social distinction, there is a chance that people "misattribute" (Webster and Hysom 1998:357) apparent worth and competence differences to differences in the characteristic. The likelihood that this happens depends on the consensus in the mutual respect and competence evaluations that individuals perceive (Ridgeway 2000; Ridgeway and Correll 2006). Using Ridgeway's words:

[. . . P]eople take on status beliefs from interaction because of the appearance of consensus in a local situation that makes an enacted correspondence between people's nominal differences and [. . . competence appear to be a valid social fact. Subsequent encounters that confirm this status belief [. . .] broaden the appearance of consensus about the status belief. As the appearance of consensus is validated across contexts and actors, the belief becomes part of the actor's accepted social framework. (Ridgeway 2000:99)

Thus, the observation of hierarchical differentiation in a particular context can create status beliefs because it conveys the impression of consensual agreement in the mutual evaluations of the involved interactants. For these beliefs to be maintained, subsequent encounters with different individuals in different contexts need to sufficiently back up the perceived consensus. Once a belief appears largely consensual, a small number of deviating experiences are unlikely to undermine it (Ridgeway 2000). 


\section{Interactions and Network Clustering}

SCT highlights the importance of face-to-face, goal-oriented interactions for belief emergence and diffusion. A large body of research on the structure of social networks suggests that such interactions do not occur at random between any two members of a population, especially if the population is large. Instead, interaction patterns tend to be clustered. This means that people mainly interact with a small subset of the larger population, and members of these subsets tend to be highly interconnected with each other. This gives rise to local communities (or clusters) in the network, within which people tend to be highly interconnected but that are typically loosely connected to other communities (Watts 1999; Wong et al. 2006).

Network clustering can have many reasons. One important source for network clustering is the constraints that physical distances impose on meeting opportunities (e.g., Faust et al. 1999; Festinger et al. 1950; Mok, Wellman, and Basu 2007; Preciado et al. 2012; Sailer and McCulloh 2012; Wong et al. 2006). Interacting face-to-face with somebody who lives far away often requires costly and time-consuming travelling. Yet people's resources and time budgets are limited, and this has the effect that most of their interactions take place with people who live in close physical proximity. As a result, interaction networks tend to be clustered in physical space, so the people who live in a given geographic region share more ties among each other than with members of other geographic regions. Spatial distance has been found to matter even in the organizational context, in which arguably most everyday goaloriented interactions take place. For instance, spatial distance negatively affects the likelihood that collaboration occurs between individual scientists, companies, and companies and universities (Balland 2012; Hoekman, Frenken, and Tijssen 2010; Katz 1994; Ponds, Van Oort, and Frenken 2007). Similarly, individuals often encounter coworkers who live in the same geographic area (cf. Niles and Hanson 2003). This can be attributed to limits in the time that most people are willing to commute every day (cf. Rouwendal 1999).

Constraints on network formation that foster network clustering are not only geographical but also social in nature. Network research has identified at least three important processes that promote network clustering. First, transitive closure, the tendency of people to close triads in social networks, has been derived from social balance theory (Cartwright and Harary 1956; Heider 1946) and shown to entail the clustering of network relations. Transitive closure characterizes the structure and dynamics of networks in many contexts (e.g., Faust 2007). Second, foci (Feld 1982), like schools, neighborhoods, or workplaces, often systematically sort similar people into similar contexts where they are likely to interact more with each other than with those outside of this context. Finally, homophily (Lazarsfeld and Merton 1954; McPherson, Smith-Lovin, and Cook 2001), the principle that people more likely interact and communicate with similar others, has been identified as one of the most prevalent regularities of social life. Homophily may result from structural processes (like foci) or from a stronger attraction to more similar others (Byrne 1971) that leads similar people to preferentially seek each other as partners of interaction (e.g., Pearson, Steglich, and Snijders 2006; Stark and Flache 2012). In both cases, homophily fosters clustering of network relations among subsets of relatively similar individuals. 
The extent to which social networks are clustered can vary across populations. For example, the effect that physical distance has on interaction patterns can vary. In highly developed countries, with a high density of car ownership and public transportation, it is easier to cover a distance of 10,100, or more miles than in less developed countries, in which walking is the main mode of transportation for a large part of the population (cf. Mok et al. 2007). Moreover, the accessibility of communication infrastructures, such as internet and telephone networks, can differ substantially between societies (cf. World Bank 2017). Similarly, the extent to which social sources of network closure, such as transitive closure or homophily, affect the formation of networks depends on contextual conditions, such as the size or heterogeneity of groups or different social norms about friendship formation (e.g., Goodreau, Kitts, and Morris 2009).

Network clustering is therefore best conceptualized as a continuum. If clustering is low, any two members of a population are equally likely to maintain a tie with each other. If clustering is high, individuals are mostly connected to people who are connected to a similar set of the population so that tightly knit communities emerge. At intermediate levels, individuals are part of local communities but also maintain some ties with people who belong to other communities. Such long-ranging ties tend to create so-called "small-world structures" (Watts and Strogatz 1998), in which most people are connected within local communities but still are indirectly connected to rest of the population through only a few steps in the network.

\section{The Effects of Network Clustering on Status Construction}

We expect that network clustering can constrain the diffusion of status beliefs and can even give rise to diversity in status beliefs if belief inertia is considered. Our expectation follows from a three-step process of belief formation and diffusion.

First, we expect that communities are likely to differ in the status beliefs that spontaneously develop among their members. The status hierarchy emergence assumption implies that by chance, a number of interactions in a given local community might put members of one social category in the status-advantaged position (cf. Mark et al. 2009; Ridgeway 2000). The perceived consensus and belief change assumption holds that this experience might induce a corresponding status belief in at least some community members. If there are no systematic competence differences between members of the different categories, these chance processes will favor each category with equal probability (cf. Grow et al. 2015; Mark et al. 2009; Ridgeway 2000). In large populations, many interactions take place simultaneously in different communities. It is therefore likely that members of one category are advantaged in some communities but are disadvantaged in others.

Second, we expect that a spontaneously created status belief is likely to induce consistent interactional experiences in the community in which it has been created and thereby will become consensual among community members. The status belief effect assumption holds that individuals are likely to treat their interaction partners in line with their status beliefs. In highly clustered communities, interaction networks are very dense. This means that as soon as at least some community members have acquired a status belief and treat others accordingly, many more 
community members will be exposed to similar interactional experiences in favor of one category. This creates the impression of consensus, even among those who have not acquired a corresponding belief yet. This, in turn, renders them likely to actually acquire the belief and ultimately leads to widespread consensus in the community.

Third, we expect that once an actual consensus on a status belief has been reached among the members of a local community in the network, this consensus is likely to ward off influence from interactions with members of other communities in which alternative status beliefs might have merged. The perceived consensus and belief change assumption holds that status beliefs are maintained as long as they are perceived as sufficiently consensual. When network clustering is high, interaction networks are dense within communities but sparse between communities. As a consequence, once a status belief actually has become consensual in a given community, members of this community will be repeatedly exposed to the same interactional experiences. This is likely to reinforce the impression of consensus, which, in turn, can ward off social influence from occasional interactions with members of other communities, in which other status beliefs might have emerged.

The foregoing process depends on the fact that people can become resistant to belief change if their current belief appears largely consensual in their immediate social environment. If a few (or even a single) contradicting experiences would be sufficient for people to lose a belief that appears largely consensual in their immediate social environment, it is unlikely that different locally consensual status beliefs could persist in different regions of the network, even if networks are clustered.

\section{The Formal Model}

In this section, we first describe the model proposed by Mark et al. (2009) and subsequently discuss our extensions.

\section{The Original Model}

The model consists of a population of $I$ actors. Each individual actor $i$ is characterized by a social distinction $N_{i}$ and a status belief $S_{i}$. The social distinction has the two states $A$ and $B\left(N_{i} \in\{A ; B\}\right)$, representing a salient social characteristic with two categories, such as gender with the categories male and female or race with the categories black and white. These states are fixed and visible to other actors. The status belief has three states: $A, O$, and $B\left(S_{i} \in\{A ; O ; B\}\right)$. These states are flexible and can change over time, but they are not visible to other actors. When $S_{i}=A$ or $S_{i}=B$, actor $i$ believes that those with the corresponding state on $N_{i}$ are more respected and competent than actors with the respective other state; when $S_{i}=O$, actor $i$ does not believe that the members of the different categories differ in worth and competence.

Actors engage in small group interactions to reach collective goals. The model focuses on the dyad as the smallest possible group, and actors are randomly paired with another member of the population to engage in dyadic interaction. During 
any interaction, a hierarchy can emerge that puts one actor (say actor $i$ ) in a statusadvantaged (i.e., more influential) position, and the other actor (say actor $j$ ) in a status-disadvantaged (i.e., less influential) position. When the actors belong to the same social category so that they share the same state on $N_{i}$, there is nothing that might favor either of the two actors to take the advantaged position. Still, in line with the status hierarchy emergence assumption, a hierarchy can emerge spontaneously with probability $h(0 \leq h \leq 1) .{ }^{1}$ If this happens, both actors are equally likely to take the status-advantaged or the status-disadvantaged position.

The situation is more complex when the actors belong to different categories, especially when one of the interaction partners believes that members of one category are more worthy and competent than members of the other category. Table 1 illustrates how $S_{i}$ and $S_{j}$ can combine in dyads whose members differ in $N_{i}$. It also shows the probabilities with which different types of status hierarchies can emerge. Consider first situations in which either $i$ or $j$ holds a status belief, whereas the respective other actor holds no belief, or in which both actors hold the same belief (cells 1, 2, 4, 6, 8, and 9 in Table 1). In these cases, at least one actor is assumed to be more respectable and competent than the other by at least one member of the dyad, whereas the other member of the dyad holds at least no contrary belief. In line with the status belief effect assumption, the model assumes that an opposition-free belief affects the interactions between the actors, so it is certain that a hierarchy emerges in which the actor who is advantaged by the belief will take the higher-status position. Consider next situations in which $i$ and $j$ hold no or opposing beliefs (cells 3, 5, and 7 in Table 1). In these cases, $i$ 's and $j$ 's beliefs do not unambiguously imply who is more respectable and competent and therefore should take the higher-status position. Yet, in line with the status hierarchy emergence assumption, the model assumes that nevertheless, a hierarchy might emerge between them with probability $h$. If this happens, both actors are equally likely to take the status-advantaged or the status-disadvantaged position.

In line with the perceived consensus and belief change assumption, interactions between members of the different categories of $N_{i}$ can lead to the emergence of status beliefs among the actors. Yet, in contrast with the perceived consensus and belief change assumption as outlined above, the process of belief emergence and maintenance as modelled by Mark and his coauthors only considers one interactional experience instead of multiple experiences. After each interaction between two actors who differ in $N_{i}$, both actors update their status beliefs on the basis of the experience they have made with their interaction partner. For example, if the interaction would have been of the type shown in cell 4 of Table 1, a hierarchy would have emerged that favored members of category $A$, and therefore it would have supported the belief $S_{i}=A$ because actor $i$ belonged to this category and actor $j$ believed that members of this category are worthier of respect and more competent than members of category $B$.

Actors consider such experiences in the following way. First, if at the beginning of an interaction actor $i$ holds no belief $\left(S_{i}=O\right)$ but experiences a hierarchy in which a member of category $A$ or $B$ attains the higher-status position, $i$ will acquire a corresponding status belief with probability $a(0<a \leq 1)$. If no hierarchy emerges, the actor will not acquire any belief. Second, if at the beginning of an interaction 
Table 1: Possible combinations of status beliefs $\left(S_{i}\right)$ and outcomes of interactions in dyads whose members belong to different social categories $\left(N_{i} \neq N_{j}\right)$.

\begin{tabular}{lllll}
\hline & $\begin{array}{l}N_{j}=B \\
S_{j}=A\end{array}$ & & $\begin{array}{l}N_{j}=B \\
S_{j}=O\end{array}$ &
\end{tabular}

Note: $R_{i}$ and $R_{j}$ represent the relative status rank that the actors have attained during their interaction. For example, if $R_{i}>R_{j}$, actor $i$ was in the status-advantaged position and actor $j$ was in the status-disadvantaged position. If $R_{i}=R_{j}$, no hierarchy has emerged.

actor $i$ holds a belief $\left(S_{i}=A\right.$ or $S_{i}=B$ ) but makes an experience that contradicts this belief (e.g., no hierarchy emerges or a member of the category that $i$ believes to be less respected and competent manages to attain the high-status position), the actor loses his or her current belief with probability $l(0<l \leq 1)$. Third, if an actor makes an experience that is congruent with his or her current state on $S_{i}, S_{i}$ remains unchanged.

Note that interactions with actors who belong to the same category as $i\left(N_{i}=N_{j}\right)$ have no effect on $i$ 's beliefs. The reason is that even if hierarchies might form in such interactions, they cannot provide any information about the competence of the members of the different social categories.

\section{Model Extensions}

Our first adjustment of the model concerns the way in which actors are selected for interaction. In the original model, actors are randomly paired with other members of the population to engage in goal-oriented, dyadic interaction. However, this is in contrast with the empirical observation that interactions often occur in clustered network structures. Here we impose such a structure on the actor population. We adapt a network-generation algorithm proposed by Wong et al. (2006) for our purposes to systematically vary network clustering. In this algorithm, variation in spatial clustering of social relations is considered the most important source of network clustering, especially in large, geographically distributed populations. As Wong et al. (2006) showed, their method implies that spatial clustering is closely tied to network clustering, and the generated networks also exhibit further structural properties generally observed in empirical social networks. We can thus consider 
these spatially clustered networks as a more general model of clustered social networks.

Following Wong et al. (2006), our method assumes that actors are located in physical space and are embedded in a social network structure that is affected by the spatial distances between them. This network determines which actors can engage in dyadic interactions with each other, so only those who share a tie can interact. Technically, we create the network structure in a simple two-step process. In step one, we randomly assign actors a place of residence on a plane of size $W \times W$. In step two, we generate a binary, undirected network among the actors, in which a given pair $i$ and $j$ can either share a tie $\left(x_{i j}=1\right)$ or not $\left(x_{i j}=0\right)$. To establish the network, actors are selected one at a time (without replacement) to choose a number of

$$
k \in\{1,2, \cdots,\lfloor 0.5(I-1)\rfloor\}
$$

other actors to whom they are not connected yet for establishing a tie. The likelihood that actor $i$ will select actor $j$ from the set of available alternatives depends on the Euclidian distances between their places of residence $\left(d_{i j}\right)$ and is proportional to the value of the spatial distance function $f\left(y, d_{i j}\right)$ over all available alternatives. This function is defined as

$$
f\left(y, d_{i j}\right)=\exp \left(-y\left[d_{i j}\right]\right),
$$

in which $y(0 \leq y \leq \infty)$ governs the effect that spatial distance has on the probability that actor $i$ selects actor $j$ for establishing a tie. For a given level of $k$, when $y=0$, the network has a random structure that is not associated with spatial distances between the actors and that does not show any clustering. Increasing $y$ reduces the average distance that ties cover. This means that network clustering typically increases as $y$ increases because actors who live close to each other will increasingly be connected to the same set of other actors who also live close by. Figure 1 illustrates this for the parameter combination $k=5$ and $y=8$, which we use in our simulation experiments (see details below). ${ }^{2}$ More generally, our network construction method imposes a close link between network clustering and the value $y$, as we show in detail in the online supplement, both for the overall networks we generate and for their subnetworks that contain only the links between agents who belong to different categories.

Our second adjustment concerns the way in which actors consider their interactional experiences with those who differ from them in $N_{i}$ when they update their status beliefs. In the original model, actors update their beliefs after each interaction with a member of a different category and only consider their experience during this interaction. We extend this implementation to incorporate that people consider multiple experiences with different others and only adopt and maintain a given belief when they have the impression that it is consensual in their social environment. We implement this notion by endowing actors with a memory $M_{i}$ that contains information about their most recent experiences with all members of the opposite category with whom they have interacted so far. For example, imagine that actor $i$ belongs to category $A$ and has interacted with the actors $j, o$, and $p$ who belong to category $B$. Imagine further that $i$ 's last interactions with $j$ and $o$ supported the 


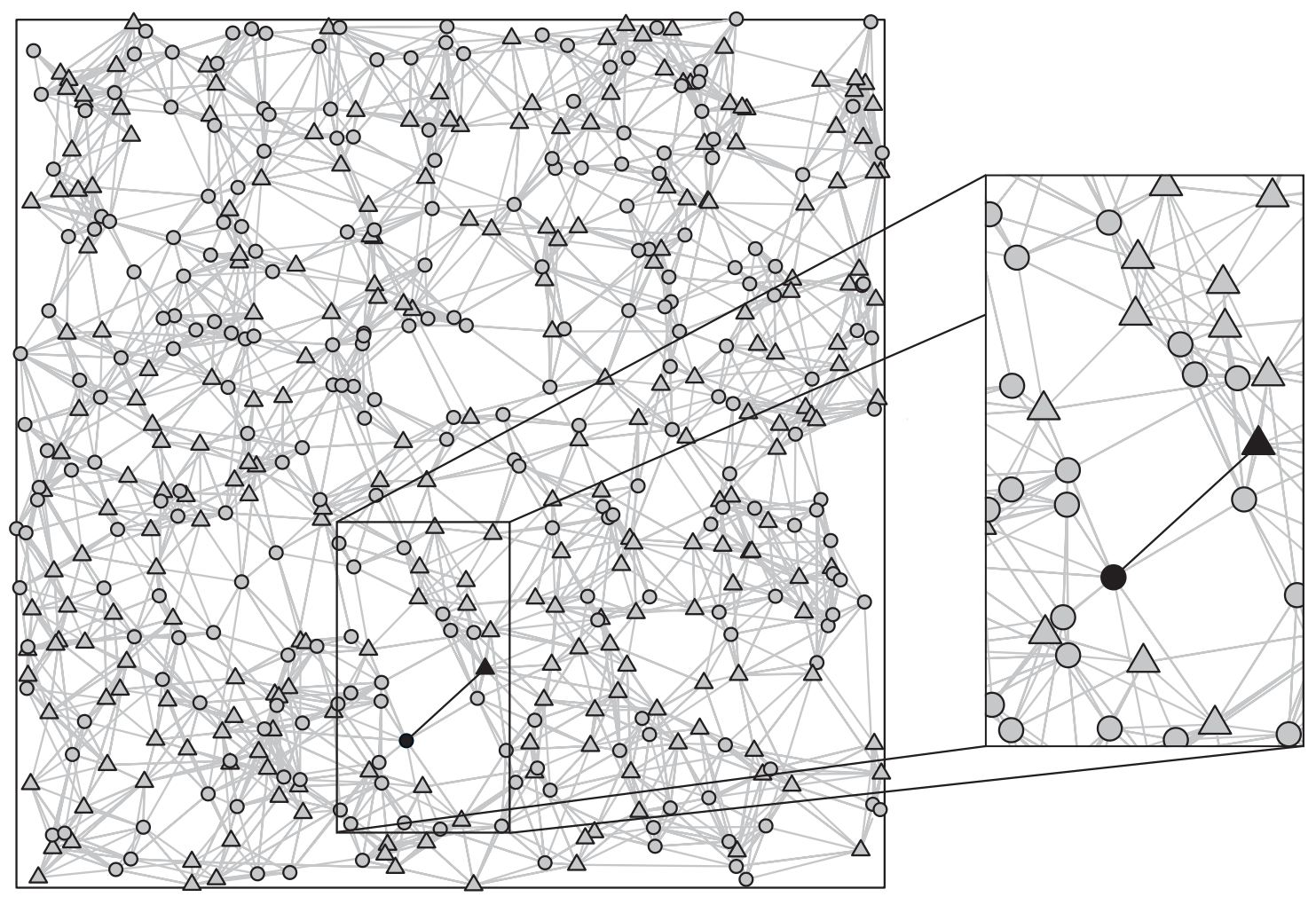

Figure 1: Example of network clustering with detailed view. Triangles and circles represent actors with $N_{i}=A$ and $N_{i}=B$, respectively. Lines between actors indicate the possibility for interactions between them. The two actors indicated with black color belong to two different communities whose members are tightly interconnected but who maintain fewer ties with members of the respective other community.

belief $B$, whereas the last inaction with $p$ did not support any belief. Hence, $M_{i}$ is filled with three elements so that $M_{i}=\{B ; B ; O\}$. These experiences are sorted by the interaction dyad to which they pertain, so the first element refers to $i$ 's last interaction with $j$, the second to the last interaction with $o$, and the third to the last interaction with $p$. This implies that the length of $M_{i}$ increases if $i$ interacts with somebody with whom he or she has never interacted before. Existing experiences remain unaffected if $i$ interacts with somebody new, but they can be updated by new experiences with past interaction partners (e.g., the third element in the above example might change if $i$ interacts again with $p$ ).

As in the original model, actors update their status beliefs after each interaction with somebody who differs from them in $N_{i}$. During this update, they use the information in $M_{i}$ to determine which belief appears most consensual in their environment. If they currently hold no belief $\left(S_{i}=O\right)$, they adopt the belief $S_{i}=A$ or $S_{i}=B$ with probability $a$ when either $A$ or $B$ accounts for the majority (i.e., $>50$ percent) of their experiences. If they currently hold a belief $\left(S_{i}=A\right.$ or $\left.S_{i}=B\right)$, but their experiences in support of this belief do not account for the majority of all their experiences in $M_{i}$ anymore, they lose this belief with probability $l$. For example, when $M_{i}=\{B ; B ; B ; A\}$, the belief $S_{i}=B$ appears consensual from $i$ 's point of 
view. Hence, if the actor currently holds no status belief, he or she will acquire this belief with probability $a$. By contrast, if $M_{i}=\{B ; B ; O ; A\}$, the belief $S_{i}=B$ would not appear sufficiently consensual and $i$ would lose this belief with probability $l$ if he or she currently held it. Thus, as in the original model, actors do not directly communicate their beliefs to each other. Instead, actors are influenced by the degree of consensus that exists in their network neighborhood via the interactional experiences with their network neighbors because every experience results not only from a given actor's own beliefs but from the combination of the beliefs of both partners involved in the interaction.

Note that if actors have so far interacted with only one other actor who differs from them in $N_{i}$, their memory will contain exactly one element. Hence, in line with the assumptions of SCT, interactional experiences in a given local context might induce a status belief, but for this belief to be maintained, subsequent interactions need to support it. Once an actor has made many congruent experiences, a single disconfirming experience is not enough to undermine an existing belief. Note also that, as in the original model, actors who currently hold a status belief that is not sufficiently supported anymore always need to transition through $S_{i}=O$ before they can adopt a new belief.

\section{Analytical Approach}

Mark et al. (2009) modelled status belief dynamics with a system of difference equations, determining expected long-run behavior of their system with an analytical solution. Their model implies that there are only two possible outcomes in the long run: either all members of the population adopt the belief $A$ or all members adopt the belief $B .{ }^{3}$ This approach tends to become intractable when heterogenous network structures need to be considered (Gilbert 2008; Macy and Flache 2009; Van Bavel and Grow 2016). To address this problem, we relied on agent-based computational modelling, which can accommodate heterogenous network structures more easily. For this, we have implemented the model in computer code and let populations of artificial actors interact with each other according to the rules outlined above. We have implemented the model in NetLogo, version 6.0.1 (Wilensky 1999). The model code can be obtained from https://www.openabm.org/model/5493, together with a more technical model description.

To assess our main argument, we added our different model adjustments in a stepwise fashion and explored how each addition affected model outcomes. That is, we started with a simulation in which we replicated the model of Mark et al. (2009). This served as a baseline and made it possible to assess whether the conclusions that we can draw from our computational model are consistent with the conclusions of Mark et al.'s (2009) difference equation model. Next, we added belief inertia to explore how it affects model outcomes on its own. Finally, we added network structures to the model and varied the level of network clustering to see how it interacts with belief inertia.

Throughout our main simulation runs, we assumed that there were 250 actors who belonged to category $A$ and 250 actors who belonged to category $B$. These actors inhabited a world of size $5 \times 5$ spatial distance units $(W)$. At the beginning 
of a given simulation run, no actor held a status belief (i.e., $S_{i}=O$ for all actors). In the stepwise procedure described above, we fixed the likelihoods that hierarchies emerged spontaneously $(h)$, that actors acquired beliefs $(a)$, and that they lost beliefs $(l)$ at an intermediate value of 0.5 . To create clustered networks, we set the number of ties that actors established $(k)$ to 5 and set the spatial distance effect $(y)$ to 8 when network structures with clustering were considered. We also considered networks that were unaffected by spatial distances, for which we employed $k=5$ and $y=0$. In two additional sets of simulation runs, we assessed whether our results might depend on the precise parametrization of the model. In particular, we assessed how much our results depended on the values of $h, a$, and $l$, and we also assessed how much our results depended on precisely how much consensus actors needed to perceive in their social environment to acquire beliefs and to become resistant to belief change. We describe the details of this sensitivity analysis after the results of our main analysis.

A given simulation run started with initializing the actor population and establishing the interaction network among them if a network was required. After this, the actors started to interact with each other so that they were selected one at a time to be paired with one of the other actors with whom they shared a tie (if there was a network) or with a randomly selected member of the population (if there was no network). During each such interaction, it was determined whether a hierarchy emerged between the actors. If the actors differed in their states on $N_{i}$, they both updated their memory with the outcome of their interaction and got the opportunity to update their status beliefs on the basis of this. They were then unpaired and the next pair was selected for interaction. ${ }^{4}$

Note that we assumed that network structures do not change over the course of a simulation run. Note also that during the network generation process, it was possible that by chance, at least one actor was not connected to somebody who differed from him or her in $N_{i}$. Given that in the model interactions with members of a different category are the only source of status beliefs, we randomly rewired one tie of actors who did not have at least one network neighbor who differed from them in $N_{i}$ to another actor who differed from them in $N_{i}$. On average, this happened for a small fraction $(1.15 / 500=0.0023)$ of all actors included in the simulation runs that considered network structures. It was also possible that a network consisted of two or more components that were not connected to each other. In this case, beliefs can by definition not diffuse throughout the entire population. However, this situation never occurred in the simulation runs that underlie our results. Finally, if there was a network, the maximal length of actors' memory was determined by the number of actors who differed from them in $N_{i}$ and with whom they shared a tie. If there was no network, actors' memory could contain as many elements as there were other actors who differed from them in $N_{i}$ in the population given that they could potentially interact with each of them.

Mark et al. (2009) showed analytically that in their model, situations in which all actors hold the belief $S_{i}=A$ or in which all actors hold the belief $S_{i}=B$ are basins of attraction. This means that once such a state has been reached, the belief configuration remains in the respective state because no actor can be induced to change his or her belief anymore. In our extended version of the model, it is also 
possible that the belief configuration reaches a stable equilibrium in which no actor can be induced to change his or her belief anymore. Yet, in contrast to Mark et al.'s (2009) model, such an equilibrium can be reached even when there is diversity in status beliefs, so some agents hold the belief $S_{i}=A$, whereas others might hold the beliefs $S_{i}=B$, or even hold no belief at all $\left(S_{i}=O\right)$. The reason for this is related to our main argument. Different status beliefs might emerge in different communities, and the interactions among the members of these communities might reinforce their beliefs so that they cannot be affected anymore by interactions with members of other communities in which different beliefs have emerged. For example, two actors $i$ and $j$ who can interact with each other and who hold different status beliefs (say, $S_{i}=A$ and $S_{j}=B$ ) may nonetheless be unable to affect each other's beliefs if they are members of different communities whose members share their respective beliefs. In such a situation, the largest shares of $i$ 's and $j$ 's interactional experiences are likely to support the beliefs they already hold, and this will make them unable to influence each other. Ultimately, this has the effect that beliefs might not diffuse across the two communities if there are only few connections between them. To ensure that differences between our results and those reported by Mark et al. (2009) do no only reflect short-term differences that might vanish if the simulation runs are continued long enough, we have developed a formal test to assess whether a given model run had reached a stable equilibrium and let each run continue until it had reached such an equilibrium. When such an equilibrium was reached, actors' status beliefs could not change anymore, even if the simulation run would have continued much longer. In the online supplement, we discuss the formal equilibrium test in detail.

Finally, because of the stochastic nature of the simulation process, we conducted 50 independent simulation runs per condition and averaged outcomes over these runs. As we also show in the online supplement, this number of simulation runs was sufficient to obtain reliable results.

\section{Outcome Measures}

We used three measures to assess the extent to which status beliefs emerged and diffused throughout the actor populations and how much this diffusion was correlated with the network structure in which the actors were embedded.

The first two measures assessed whether status beliefs emerged and how widely they diffused. The first measure is the largest share $(L S)$ of actors who either held the belief $S_{i}=A$ or the belief $S_{i}=B$. We calculated this measure as

$$
L S=\frac{\max \left(\# S_{i}=A, \# S_{i}=B\right)}{I},
$$

where $\# S_{i}=A$ and $\# S_{i}=B$ refer to the number of actors with the beliefs $S_{i}=A$ and $S_{i}=B$, respectively. $L S$ ranges from 0 to 1 . The closer it comes to 1 , the more widely a single belief has become adopted by the actors; the closer it comes to 0 , the fewer actors have adopted any status belief (i.e., the more actors hold the state $\left.S_{i}=O\right)$. Values in between indicate that diversity in status beliefs exists. The second measure built on $L S$ and assessed whether in a given run a belief had 
emerged and had been adopted by all actors, which is indicated by $L S=1$. We refer to this as complete diffusion $(C D)$, meaning that $C D=1$ when $L S=1$ and $C D=0$ when $L S<1$. If our main argument is correct, the average values of $L S$ and $C D$ should decrease when network clustering and belief inertia are considered.

Our third measure assessed a different implication of our main argument. We contend that if actors are embedded in a network structure and if this structure is clustered, those actors who are linked with each other should have a higher likelihood of holding the same state on $S_{i}$ than actors who are not linked with each other. To assess how much having the same belief was associated with being connected or not, we adapted a network segregation measure developed by Moody (2001). Moody (2001) was interested in assessing how similarity and dissimilarity in race affects the formation of friendship ties between pupils in U.S. school contexts. For this, he devised a segregation index that assessed whether pupils who belong to the same race are more likely to share a tie than pupils who belong to different races. In our case, we wanted to assess how the presence or absence of a tie between actors affected whether they held similar status beliefs. Hence, we adjusted Moody's (2001) measure so that it captures the degree to which actors who shared a tie were more likely to hold the same belief than actors who did not share a tie. We calculated this measure of network belief segregation (NBS) as

$$
N B S=\log \left[\frac{\#\left(S_{i}=S_{j} \mid x_{i j}=1\right) / \# x_{i j}=1}{\#\left(S_{i}=S_{j} \mid x_{i j}=0\right) / \# x_{i j}=0}\right] .
$$

In Equation (3), the numerator focuses on the set of all pairs of actors who share a tie and measures the fraction of these pairs that hold the same state on $S_{i}$. The denominator focuses on the set of all pairs of actors who do not share a tie and indicates the fraction that hold the same state on $S_{i}$. NBS can take values between $-\infty$ and $\infty$. A value of 0 is obtained if similarity in status beliefs is disassociated from the network structure. This can happen when there is population-wide consensus in status beliefs but also when different beliefs exist and are randomly distributed across the network. Values larger than 0 indicate positive belief clustering in the network, so actors who share a tie are more likely to hold the same state on $S_{i}$ than actors who do not share a tie. Values smaller than 0 indicate negative belief clustering in the network, meaning that actors who share a tie are less likely to hold the same state on $S_{i}$ than actors who do not share a tie. Negative belief clustering was unlikely to occur in our simulations. Evidently, NBS can only be calculated if actors are embedded in a network structure, and we thus report this measure only for conditions in which networks were present.

Furthermore, we also assessed the time it took a given simulation run to reach a stable equilibrium. For computational efficiency, we assessed convergence after every 20,000 dyadic interactions between actors (rather than after each such interaction). Hence, we measured the time to convergence (TTC) in the number of these assessment cycles. For example, a value of TTC $=3$ indicates that a given run had reached a stable equilibrium after three assessment cycles, which corresponds to about 60,000 interactions among pairs of actors. ${ }^{5}$ 


\section{Results}

\section{Results of the Main Experiment}

Table 2 shows the outcomes of our main simulation experiment. It displays the share of runs in which status beliefs had emerged and diffused throughout the entire population $(C D)$, the means and standard deviations of our measures of how widely beliefs had diffused exactly $(L S)$ and how much similarity in beliefs was associated with being linked in the network (NBS), and the means and standard deviations of the number of assessment cycles it took the simulation runs to reach a stable equilibrium (TTC).

The version of our model that replicates the assumptions of Mark and his coauthors also replicates their main result. In the condition in which actors only considered a single interactional experience (i.e., no belief inertia possible) and interactions could occur between any two population members (first row of Table 2), the simulation runs always ended in a situation in which a status belief had emerged that was held by all actors in the population; on average, this occurred after about 1 cycle of 20,000 dyadic interactions $(T T C=1.02)$. This is evidenced by the fact that the average values of both $C D$ and $L S$ were 1 . This did not change when we introduced belief inertia (second row of Table 2) or when actors were embedded in a (clustered) social network structure (third and fourth row of Table 2), albeit the average time that it took the simulation runs to reach a stable equilibrium increased, especially when belief inertia was not possible and network structures were present but not clustered (fourth row of Table 2, TTC $=321.04$ ). ${ }^{6}$ Thus, as expected, neither of our changes at the microlevel and the macrolevel of the original model were able to affect the ultimate outcome of the belief diffusion process on its own, even though it took the diffusion process much longer to generate this outcome, especially when it occurred in network structures.

The results changed drastically when belief inertia was possible and when actors were at the same time embedded in social network structures. Consider first the situation in which there was a network that was not clustered (fifth row of Table 2). In this condition, only about 28 percent of the simulation runs had ended in a situation in which a status belief had emerged and diffused throughout the entire population $(C D=0.28)$. On average, the largest share of actors who held the same state on $S_{i}$ was about 0.73 in this version of the model $(L S=0.73)$, meaning that some diversity in status beliefs emerged and persisted in the actor populations. This diversity was not much correlated with the network structure given that NBS was 0.14 (rather low). This changed when networks were clustered (sixth row of Table 2). In this condition, there was not a single run in which a belief had emerged and diffused throughout the entire population $(C D=0)$. Correspondingly, the average share of agents that held the same state on $S_{i}$ decreased by about 15 percent to 58 percent $(L S=0.58)$. This larger diversity in status beliefs was also more strongly correlated with actors' positions in the social network structure, as is evidenced by an average NBS value of 0.52 . For illustration, Figure 2 shows the outcome of a simulation run with network clustering in which the final value of NBS was 0.58. In this particular simulation run, a situation emerged in which there where several regions in the network in which actors held the same state on $S_{i}$ but who 
Table 2: Results of the main simulation experiment.

\begin{tabular}{lcccccc}
\hline Interaction & \multirow{2}{*}{$\begin{array}{c}\text { Belief } \\
\text { regime }\end{array}$} & $\begin{array}{c}\text { Network } \\
\text { inertia }\end{array}$ & clustering & & & \multicolumn{2}{c}{$L S$} & NBS & TTC \\
\cline { 5 - 7 } Random & No & - & 1 & $1(0)$ & - & $1.02(0.14)$ \\
& Yes & - & 1 & $1(0)$ & - & $18.60(49.38)$ \\
Network & No & No & 1 & $1(0)$ & $0(0)$ & $2.16(0.51)$ \\
& & Yes & 1 & $1(0)$ & $0(0)$ & $321.04(642.01)$ \\
& Yes & No & 0.28 & $0.73(0.22)$ & $0.14(0.12)$ & $3.70(2.11)$ \\
& & Yes & 0 & $0.58(0.07)$ & $0.52(0.07)$ & $2.88(0.85)$ \\
\hline
\end{tabular}

differed in this state from the actors in adjacent regions. In some regions, the belief $S_{i}=A$ had emerged and had become locally consensual. Yet in other regions, the belief $S_{i}=B$ had emerged and become consensual. Occasionally, there were actors who were connected to members of different regions and whose experiences were not sufficiently consistent to induce a status belief $\left(S_{i}=O\right)$. Generally, in both conditions, the resulting equilibria were reached comparatively quickly, as indicated by average TTC values of 3.70 and 2.88 for the conditions without and with network clustering, respectively.

Taken together, our results largely support our main argument. If actors exhibit belief inertia, diversity in status beliefs can emerge and persist but only when interactions between actors are constrained by social network structures. This is most likely when the network structure is clustered, but, unexpectedly, some level of diversity in status beliefs can emerge and persist even when networks are not clustered. The reason is that even when networks are not clustered, they constrain interaction possibilities. It can therefore happen that at least one subset of the population is in a stable configuration that supports belief $S_{i}=A$, whereas other subsets of the population are in stable configurations that support belief $S_{i}=B$ (or vice versa).

\section{Results of the Sensitivity Analysis}

The results of our main simulation experiment support our argument, but how much does the emergence and persistence of diversity in status beliefs depend on the likelihood that hierarchies emerge spontaneously $(h)$, that agents adopt a status belief that appears consensual $(a)$, or that agents lose beliefs that do not appear consensual anymore $(l)$ ? To answer this question, we focused on the version of the model in which interaction networks were clustered and in which belief inertia was possible (sixth row of Table 2), and we varied $h, a$, and $l$ each in three steps $(0.25$, 0.5 , and 0.75). We conducted 50 independent simulation runs for each of the 27 possible parameter combinations. Figure 3 shows the average values of $L S$ and $N B S$ that we obtained for each combination (the average value of $C D$ was 0 in all conditions). The figure suggests that the exact values of the three parameters did not affect our conclusion substantially; also, the average time to convergence $(T T C=3.40$ across all runs in this part of the sensitivity analysis) did not vary 


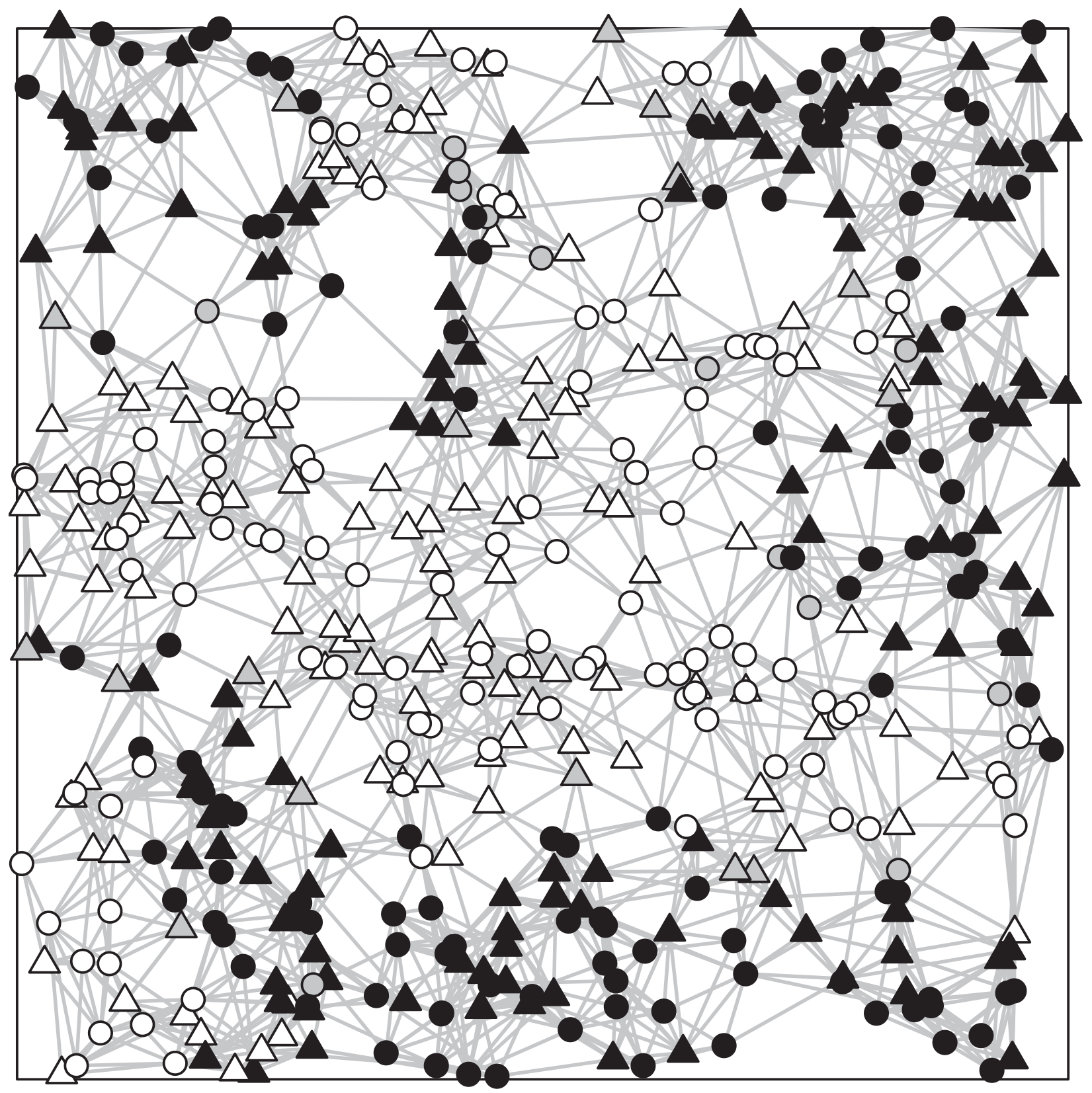

Figure 2: Example with network clustering and belief inertia. Outcomes of a typical simulation run in the main experiment that ended with diversity in status beliefs $(L S=0.49)$ and that was associated with the network structure $(N B S=0.58)$. Triangles and circles represent actors with $N_{i}=A$ and $N_{i}=B$, respectively. Lines between actors indicate the possibility for interactions between them. White actor coloring indicates that the actor holds the belief $S_{i}=A$, gray coloring indicates $S_{i}=O$, and black coloring indicates $S_{i}=B$. The parameter setting was $I=500$ (equally split into actors with $N_{i}=A$ and $N_{i}=B$ ), $W=k=5, y=8$, and $h=a=l=0.5$. The stable equilibrium was reached after four assessment cycles $(T T C=4)$. 

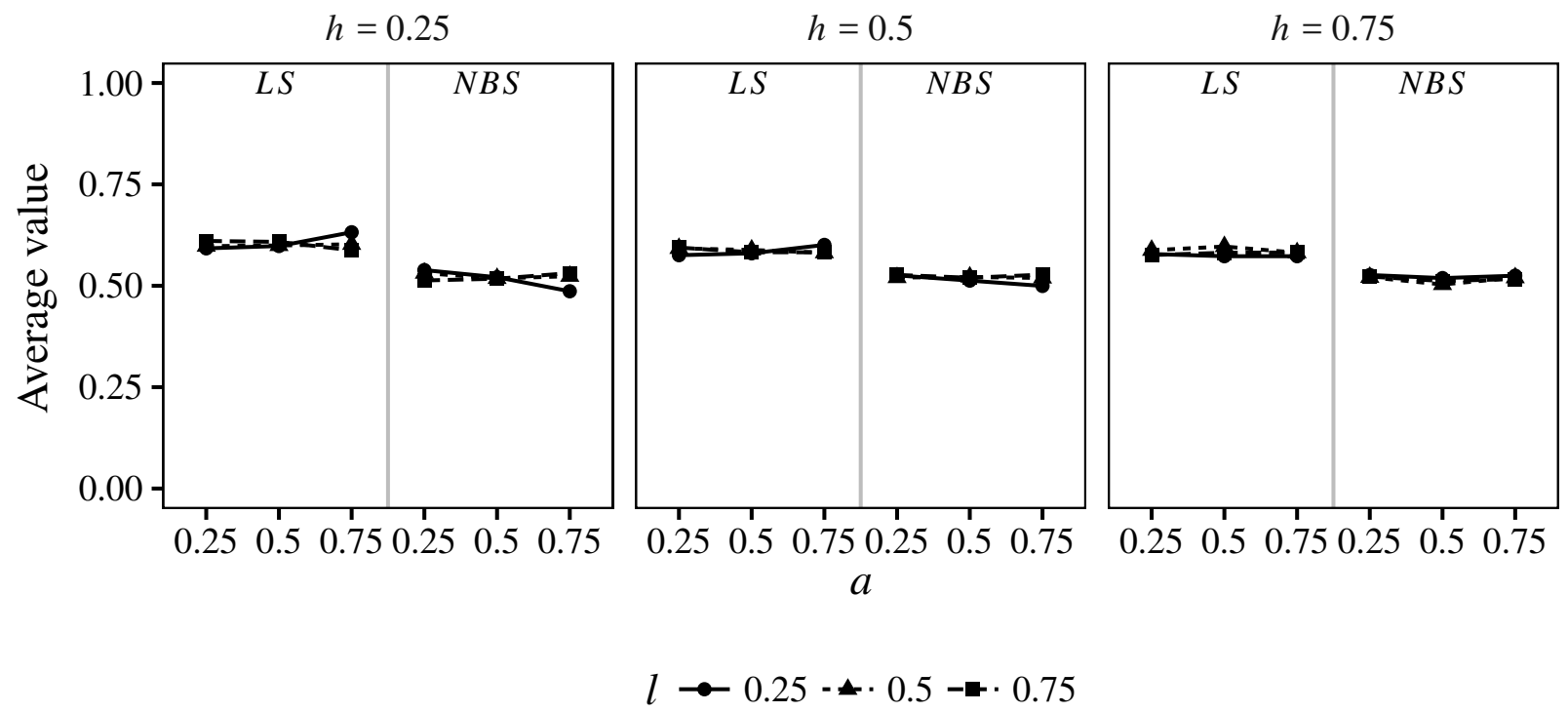

Figure 3: Results of a sensitivity analysis that focused on how much the parameters $h, a$, and $l$ affect simulation outcomes when clustered networks and belief inertia are considered.

much across the different parameter combinations, with a minimum of TTC $=2.56$ for $h=0.5, a=0.5$, and $l=0.75$, and a maximum of $T T C=4.48$ for $h=0.75$, $a=0.25$, and $l=0.25$. That is, even though there was some small variation in the exact values of $L S$ and NBS that we obtained and in the time it took the simulation runs to reach a stable equilibrium, it was the case in all conditions that diversity in status beliefs emerged and persisted and that differences in beliefs between actors were associated with differences in their position in the network structure.

Next, we assessed how much our results depended on the precise level of consensus that was needed for belief acquisition and maintenance. In our main experiments, we assumed that belief inertia was possible when a given belief $\left(S_{i}=A\right.$ or $S_{i}=B$ ) was supported by the majority of a given actor's experiences (i.e., accounted for more than 50 percent of all experiences in his or her memory $M_{i}$ ). In a third set of simulation runs, we increased this threshold to 70 percent and 90 percent and again conducted 50 independent simulation runs per condition. In these runs, we assumed that $h=a=l=0.5$, and we assumed that the interaction networks among the actors were clustered (similar to the sixth row of Table 2). Table 3 shows the results of this set of simulation runs. It suggests that increasing the threshold from $>50$ percent to $>70$ percent or $>90$ percent led to an increase in the diversity in status beliefs among the actors, as evidenced by the fact that the average values of $L S$ decreased. At the same time, the correlation between the network structure and similarity in status beliefs among the actors decreased, as evidenced by a decrease in the average value of NBS. To illustrate what this decrease means, Figure 4 shows the results of a run in which more than 90 percent of actors' experiences needed to support a given belief and in which the final NBS value was close to 0.34 . In 
Table 3: Results of a sensitivity analysis that focused on how much the precise level of consensus that is needed for belief acquisition and maintenance affects model outcomes.

\begin{tabular}{|c|c|c|c|c|}
\hline \multirow[t]{2}{*}{ Consensus needed } & \multirow[t]{2}{*}{$C D$} & LS & NBS & TTC \\
\hline & & Mean (SD) & Mean (SD) & Mean (SD) \\
\hline$>50 \%$ & 0 & $0.58(0.08)$ & $0.53(0.08)$ & $2.78(0.89)$ \\
\hline$>70 \%$ & 0 & $0.54(0.07)$ & $0.55(0.06)$ & $6.18(3.69)$ \\
\hline$>90 \%$ & 0 & $0.41(0.04)$ & $0.34(0.06)$ & $343.66(564.88)$ \\
\hline
\end{tabular}

this particular simulation run, there was some diversity in status beliefs, and this diversity was associated with actors' positions in the network, but this association was weaker than in the example shown in Figure 2. There were also more actors who did not hold any status belief. This illustrates than when belief acquisition and maintenance required very high levels of local consensus, status beliefs were generally less likely to emerge. Still, when beliefs emerged, they were likely to be clustered in the network so that actors who maintained ties to a similar set of other actors were more likely to acquire the same belief. Generally, when the threshold increased, the average time it took runs to reach a stable equilibrium also increased (from $T T C=2.78$ for a threshold of $>50$ percent to $T T C=343.66$ for a threshold of $>90$ percent). ${ }^{7}$ The reason is that for agents who are located at the intersection between two communities whose members have developed different status beliefs, it is easier to obtain a situation in which, say, slightly more than 50 percent of their neighbors hold the same belief (which makes them resistant to belief change) than it is to obtain a situation in which more than 90 percent of their neighbors hold the same belief.

\section{Discussion and Conclusion}

In this article, we have proposed an extension of the formal elaboration of status construction theory (SCT) developed by Mark et al. (2009). Whereas their earlier modelling work suggests that the processes that SCT describes have a strong tendency to generate a population-wide consensus in status beliefs, the results of our simulation experiments point to new implications of formalizing SCT.

Our results show that different status beliefs can emerge and persist next to each other. We show how this depends on the combination of a macrostructural property of the network of interaction possibilities between population members and an extension of the microprocess of belief acquisition and belief loss. Diversity of beliefs can emerge and persist under SCT dynamics when actors exhibit belief inertia and when the face-to-face interactions that can generate status beliefs are constrained by social network structures. Persistent diversity in status beliefs is most likely when network structures are clustered, but it can even occur when there is no clustering.

The model by Mark et al. (2009) was designed to explain the emergence of status beliefs with a minimal set of assumptions based on SCT. We have changed this model as little as possible to assess the logical consistency of our argument. 


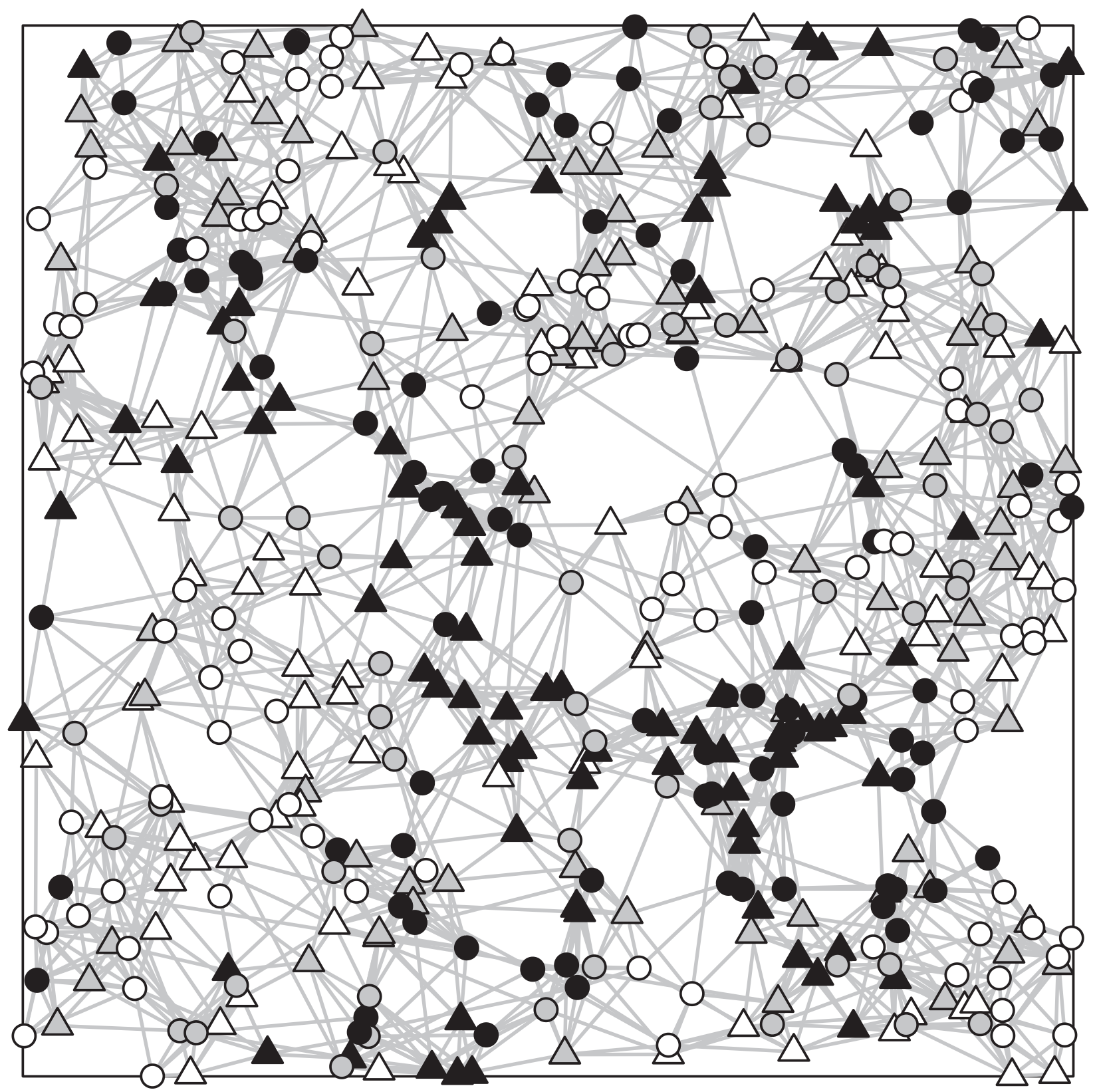

Figure 4: Example with network clustering, belief inertia, and high requirements for consensus. Outcomes of a typical simulation run in the sensitivity analysis in which actors required high consensus in their experiences for belief acquisition and maintenance ( $>90$ percent) that ended with diversity in status beliefs $(L S=0.36)$ and that was associated with the network structure $(N B S=0.34)$. Triangles and circles represent actors with $N_{i}=A$ and $N_{i}=B$, respectively. Lines between actors indicate the possibility for interactions between them. White actor coloring indicates that the actor holds the belief state $S_{i}=A$, gray coloring indicates $S_{i}=O$, and black coloring indicates $S_{i}=B$. The parameter setting was $I=500$ (equally split into actors with $N_{i}=A$ and $\left.N_{i}=B\right), W=k=5, y=8$, and $h=a=l=0.5$. The stable equilibrium was reached after 72 assessment cycles $(T T C=72)$. 
Thus, our extended model abstracts from other factors that might also affect the emergence and diffusion of status beliefs in real life. Future research that integrates such aspects into the model might produce intriguing new insights.

One important factor that the model excludes is resource and power differences between members of the different social categories. Such differences often exist in real life and can vary geographically. Status construction theory explicitly allows for the inclusion of resource differences between members of different social groups. In an early formulation of the theory, Ridgeway (1991) stressed that salient differences in resources and power between individuals are often used as cues for inferring differences in social worth and competence (see also Ridgeway et al. 1998; Ridgeway and Balkwell 1997; Webster and Hysom 1998). What implications could this have in the light of our analysis? Imagine a population with network clustering and imagine that in some geographic regions, the members of one category are slightly resource advantaged, whereas the opposite association exists in other regions. The behavioral processes that our model describes are likely to reinforce and amplify biases in status beliefs induced by these initially small differences. The regions might therefore end up with large differences in status beliefs.

Furthermore, resource and power differences not only lead to status beliefs. Status beliefs can also lead to resource and power differences (Ridgeway 1991) because status-advantaged individuals often receive more resources from others and more easily attain positions of power (cf. Merton 1968). In the light of our model, this reversed causality has important implications for current research practice. Some earlier studies interested in explaining why the status values of some social distinctions sometimes differ across geographic regions have typically started with identifying regional variation in factors that, historically, might have led to regional variation in power and resources between social categories (e.g., Balkwell and Balswick 1981; Ishii-Kuntz and Lee 1987). Our model suggests the theoretical possibility that regional variation in the distribution of power and resources can also be preceded by regional variation in status beliefs when interactions between people mostly occur among those who live in close spatial proximity to each other. Future research might benefit from taking this possibility into account.

A second important factor that the model does not address explicitly is that social networks often cluster not only in physical space but also in social space. Although both spatial and social processes can generate very similar forms of clustering in networks, more complex structural properties may arise when spatial and social dynamics operate in tandem. For example, if people who are similar to each other in social characteristics that have meaning to them (e.g., age, sex, etc.) are more likely to interact with each other than people who are more dissimilar in such characteristics (e.g., McPherson et al. 2001), this may generate a second dimension of network clustering that can strengthen or weaken the effects of spatial clustering. Other social processes may even run against the formation of clustered structures-for example, when certain people consciously choose not to interact with some other members of the population because they are in direct competition for some valuable objects, such as popularity (cf. Pál et al. 2016). Future research could incorporate such additional drivers of network clustering and explore how this affects the relation between network clustering and diversity of status beliefs. 
Finally, in line with the model proposed by Mark et al. (2009), and also in line with other research in SCT, we focused on indirect social influence processes that derive from interactional experiences between members of different social categories. Another important source of social influence might be the direct communication of status beliefs between members of the same social categories. For example, men might communicate to other men what they believe about what women are worth and capable of. Future research might incorporate this source of status beliefs into our model and connect models of the formation of status beliefs to the broad literature on formally modelling social influence dynamics. Drawing on research on homophily and confirmation bias (Nickerson 1998), models of "similarity-biased social influence" (Flache et al. 2017) assume that influence is both stronger and more likely to occur between actors with similar characteristics (e.g., Axelrod 1997; Flache and Macy 2011a; Mark 1998, 2003). This work suggests that adding social influence between actors of the same categories can be expected to strengthen tendencies towards local clustering of status beliefs. At the same time, given the lack of research into how individuals integrate information derived from interactional experience and direct communication in the process of the formation of status beliefs, much theoretical groundwork is still needed to clarify how exactly the complex dynamics of social influence and status belief formation interact at the microlevel, leaving room for new and unexpected theoretical results.

Despite the need for future research, the results that we have presented here have important implications for the explanatory scope of status construction theory. Our analysis suggests that if we assume that people consider multiple past experiences in the belief formation process, and if the interactions that can lead to such experiences occur in social network structures, the mechanisms that SCT describes may not only produce population-wide consensus but also diversity in status beliefs.

\section{Notes}

1 The parameter $h$ was denoted $e$ in Mark et al. (2009). We have opted for $h$ to avoid that $e$ might be confused with the base of the natural logarithm.

2 Watts and Strogatz (1998) proposed a global clustering coefficient (GCC) that indicates how clustered the ties in a given network are, by assessing how cliquish the connections are that actors maintain. GCC is calculated by first determining for each actor the share of the possible ties among his or her direct network neighbors that are actually realized (the share is 0 when none of actor $i$ 's direct network neighbors share a tie with each other and 1 when all of them share ties with each other). These shares are then averaged across all actors. As we illustrate in the online supplement, for $k=5$ and $y=8$ the value of GCC was typically about 0.35 in our simulations, which has been considered high in the literature (cf. Wong et al. 2006). We also propose in the online supplement a generalization of this clustering coefficient to the (bipartite) subnetwork consisting of only the links between agents who belong to different categories. Only these links are consequential for belief formation in our model. The clustering coefficient of Watts and Strogatz (1998) is not applicable for this subnetwork, but as we show, results for the relation between spatial clustering $(y)$ and network clustering also apply to our generalized measure of clustering for the bipartite subnetwork. 
3 To be precise, if the initial distribution is exactly 50 percent $S_{i}=A$ and 50 percent $S_{i}=B$, the expected long-run behavior is that nothing changes. However, as Mark et al. (2009) point out, this is an instable equilibrium from which the dynamics would inevitably move into one of the two possible consensus states if the slightest random deviation from the initial state were to occur.

4 Note that for computational efficiency, in the actual simulation experiments the model only simulates interactions between actors who differ from each other in $N_{i}$ given that interactions between actors who belong to the same category have no effect on belief formation.

5 Note that because in the actual simulation experiments the model only simulates interactions between actors to differ from each other in $N_{i}$, TTC refers only to such interactions.

6 Note that this average value was strongly affected by a small number of runs that took very long to reach a stable equilibrium. We therefore also inspected the median time until a stable equilibrium had been reached. The median for this this condition was 81.5 , whereas in the other conditions the median values ranged from 1 to 7 , closely tracing the order of the average values. Hence, this measure also indicates that in this condition, it took the runs considerably longer to reach a stable equilibrium than in the other conditions.

7 In the condition in which $>90$ percent consensus was needed, the average value of TTC was affected by a small number of runs that required an exceptionally long time to reach a stable equilibrium. Yet when the median was considered, the time it took the simulation runs to converge in this condition was also much longer (with a median of 169 cycles) than for the other conditions (with median values of 3 for $>50$ percent consensus and 5 for $>70$ percent consensus).

\section{References}

Anderson, Cameron, Michael W. Kraus, Adam D. Galinsky, and Dacher Keltner. 2012. "The Local-Ladder Effect: Social Status and Subjective Well-Being." Psychological Science 23(7):764-71. https : //doi .org/10.1177/0956797611434537

Axelrod, Robert. 1997. "The Dissemination of Culture: A Model with Local Convergence and Global Polarization." Journal of Conflict Resolution 41(2):203-26. https : //doi .org/ $10.1177 / 0022002797041002001$

Bales, Robert F. 1970. Personality and Interpersonal Behavior. New York: Holt, Rinehart and Winston.

Balkwell, Carolyn, and Jack Balswick. 1981. "Subsistence Economy, Family Structure, and the Status of the Elderly." Journal of Marriage and Family 43(2):423-29. https : / doi . org/ $10.2307 / 351392$

Balland, Pierre-Alexandre. 2012. "Proximity and the Evolution of Collaboration Networks: Evidence from Research and Development Projects within the Global Navigation Satellite System (GNSS) Industry." Regional Studies 46(6):741-56. https://doi.org/10.1080/ 00343404.2010 .529121

Berger, Joseph, M. Hamit Fisek, Robert Z. Norman, and Morris Zelditch Jr. 1977. Status Characteristics and Social Interaction: An Expectation-States Approach. New York: Elsevier.

Berger, Joseph, David G. Wagner, and Murray Webster Jr. 2014. "Expectation States Theory: Growth, Opportunities, and Challenges." Pp. 19-55 in Advances in Group Processes, edited 
by S. R. Thye and E. J. Lawler. Bingley: Emerald Publishing. https : //doi .org/10.1108/ S0882-614520140000031000

Blau, Peter M. 1964. Exchange and Power in Social Life. New York: Wiley.

Byrne, Donn Erwin. 1971. The Attraction Paradigm. New York: Academic Press.

Carli, Linda L., Suzanne J. LaFleur, and Christopher C. Loeber. 1995. "Nonverbal Behavior, Gender, and Influence." Journal of Personality and Social Psychology 68(6):1030-41. https : //doi.org/10.1037/0022-3514.68.6.1030

Cartwright, Dorwin, and Frank Harary. 1956. "Structural Balance: A Generalization of Heider's Theory." Psychological Review 63(5):277-93. https : //doi .org/10.1037/h0046049

Centola, Damon, and Micheal W. Macy. 2007. "Complex Contagions and the Weakness of Long Ties." American Journal of Sociology 113(3):702-34. https : //doi .org/10 . 1086/ 521848

Davis, James A. 1970. "Clustering and Hierarchy in Interpersonal Relations: Testing Two Graph Theoretical Models on 742 Sociomatrices." American Sociological Review 35(5):84351. https://doi.org/10.2307/2093295

DellaPosta, Daniel, Yongren Shi, and Michael W. Macy. 2015. "Why Do Liberals Drink Lattes?" American Journal of Sociology 120(5):1473-511. https : //doi .org/10 . 1086/681254

Driskell Jr., James E. 1982. "Personal Characteristics and Performance Expectations." Social Psychology Quarterly 45(4):229-37. https : //doi .org/10.2307/3033918

Faust, Katherine. 2007. "Very Local Structure in Social Networks." Sociological Methodology 37(1):209-56. https://doi.org/10.1111/j.1467-9531.2007.00179.x

Faust, Katherine, Barbara Entwisle, Ronald R. Rindfuss, Stephen J. Walsh, and Yothin Sawangdee. 1999. "Spatial Arrangement of Social and Economic Networks Among Villages in Nang Rong District, Thailand." Social Networks 21(4):311-37. https://doi. org/10.1016/S0378-8733(99)00014-3

Feagin, Joe R. 1991. "The Continuing Significance of Race: Antiblack Discrimination in Public Places." American Sociological Review 56(1):101-16. https://doi .org/10. 2307/2095676

Feld, Scott L. 1982. "Social Structural Determinants of Similarity among Associates." American Sociological Review 47(6):797-801. https : //doi .org/10.2307/2095216

Festinger, Leon, Stanley Schachter, and Kurt Back. 1950. Social Pressures in Informal Groups: A Study of Human Factors in Housing. Stanford: Stanford University Press.

Flache, Andreas, Michael Mäs, Thomas Feliciani, Edmund Chattoe-Brown, Guillaume Deffuant, Sylvie Huet, and Jan Lorenz. 2017. "Models of Social Influence: Towards the Next Frontiers." Journal of Artificial Societies and Social Simulation 20(4).https: //doi.org/10.18564/jasss.3521

Flache, Andreas, and Micheal W. Macy. 2011a. "Local Convergence and Global Diversity: From Interpersonal to Social Influence." Journal of Conflict Resolution 55(6):970-95.

Flache, Andreas, and Micheal W. Macy. 2011b. "Small Worlds and Cultural Polarization." The Journal of Mathematical Sociology 35(1-3):146-76.

Foschi, Martha, Larissa Lai, and Kirsten Sigerson. 1994. "Gender and Double Standards in the Assessment of Job Applicants." Social Psychology Quarterly 57(4):326-39. https : //doi.org/10.2307/2787159

Fournier, Marc A. 2009. "Adolescent Hierarchy Formation and the Social Competition Theory of Depression." Journal of Social and Clinical Psychology 28(9):1144-72. https: //doi.org/10.1521/jscp.2009.28.9.1144 
Ghaed, Shiva G., and Linda C. Gallo. 2007. "Subjective Social Status, Objective Socioeconomic Status, and Cardiovascular Risk in Women." Health Psychology 26(6):668-74. https: //doi.org/10.1037/0278-6133.26.6.668

Gilbert, Nigel. 2008. Agent-Based Models. Thousand Oaks: Sage Publications. https: //doi.org/10.4135/9781412983259

Goode, William J. 1979. The Celebration of Heroes: Prestige as a Social Control System. Berkeley: University of California Press.

Goodreau, Steven M., James A. Kitts, and Martina Morris. 2009. "Birds of a Feather, or Friends of a Friend? Using Exponential Radom Graph Models to Investigate Adolescent Social Networks." Demography 46(1):103-25. https : //doi .org/10.1353/dem.0.0045

Granovetter, Mark. 1973. "The Strength of Weak Ties." American Journal of Sociology 78(6):1360-80. https://doi.org/10.1086/225469

Grow, André, Andreas Flache, and Rafael P. M. Wittek. 2015. "An Agent-Based Model of Status Construction in Task Focused Groups." Journal of Artificial Societies and Social Simulation 18(2):4. https://doi.org/10.18564/jasss.2740 https://doi.org/10.18564/ jasss. 2740

Grow, André, Károly Takács, and Judit Pál. 2016. "Status Characteristics and Ability Attributions in Hungarian School Classes: An Exponential Random Graph Approach." Social Psychology Quarterly 2(79):156-67. https : //doi .org/10.1177/0190272516643052

Heider, Fritz. 1946. "Attitudes and Cognitive Organization." The Journal of Psychology 21(1):107-12. https://doi.org/10.1080/00223980.1946.9917275

Hendrix, Lewellyn, and Zakir Hossain. 1988. "Women's Status and Mode of Production: A Cross-Cultural Test." Signs 13(3):437-53. https ://doi .org/10.1086/494427

Hoekman, Jarno, Koen Frenken, and Robert J. W. Tijssen. 2010. "Research Collaboration at a Distance: Changing Spatial Patterns of Scientific Collaboration within Europe." Research Policy 39(5):662-73. https: //doi . org/10.1016/j.respol .2010.01.012

Huo, Yuen J., Kevin R. Binning, and Ludwin E. Molina. 2010. "Testing an Integrative Model of Respect: Implications for Social Engagement and Well-Being." Personality and Social Psychology Bulletin 36(2):200-212. https : //doi .org/10.1177/0146167209356787

Ishii-Kuntz, Masako, and Gary R. Lee. 1987. "Status of the Elderly: An Extension of the Theory." Journal of Marriage and Family 49(2):413-20. https : //doi .org/10 . 2307/352310

Katz, J. Sylvan. 1994. "Geographical Proximity and Scientific Collaboration." Sociometrics 31(1):31-43. https://doi.org/10.1007/BF02018100

Lazarsfeld, Paul F., and Robert K. Merton. 1954. "Friendship and Social Process: A Substantive and Methodological Analysis." Pp. 18-66 in Freedom and Control in Modern Society, edited by M. Berger, T. Abel, and C. H. Page. New York: Van Nostrand.

Macy, Micheal W., and Andreas Flache. 2009. "Social Dynamics from the Bottom Up: AgentBased Models of Social Interaction." Pp. 245-68 in The Oxford Handbook of Analytical Sociology, edited by P. Hedström and P. Bearman. Oxford: Oxford University Press.

Mark, Noah P. 1998. "Beyond Individual Differences: Social Differentiation from First Principles." American Sociological Review 63(3):309-30. https : //doi .org/10.2307/2657552

Mark, Noah P. 2003. "Culture and Competition: Homophily and Distancing Explanations for Cultural Niches." American Sociological Review 68(3):319-45. https://doi .org/10.2307/ 1519727

Mark, Noah P., Lynn Smith-Lovin, and Cecilia L. Ridgeway. 2009. "Why Do Nominal Characteristics Acquire Status Value? A Minimal Explanation for Status Construction." American Journal of Sociology 115(3):832-62. https : //doi . org/10 . 1086/606142 
Marmot, Michael G. 2004. The Status Syndrome: How Social Standing Affects Our Health and Longevity. New York: Time Books.

McPherson, Miller, Lynn Smith-Lovin, and James M. Cook. 2001. "Birds of a Feather: Homophily in Social Networks." Annual Review of Sociology 27:415-44. https://doi. org/10.1146/annurev.soc.27.1.415

Merton, Robert K. 1968. "The Matthew Effect in Science." Science 159(3810):56-63. https: //doi.org/10.1126/science.159.3810.56

Mok, Diana, Barry Wellman, and Ranu Basu. 2007. "Did Distance Matter before the Internet? Interpersonal Contact and Support in the 1970s." Social Networks 29(3):430-61. https : //doi.org/10.1016/j.socnet.2007.01.009

Moody, James. 2001. "Race, School Integration, and Friendship Segregation in America." American Journal of Sociology 107(3):679-716. https : //doi . org/10.1086/338954

Moss-Racusin, Corinne A., John F. Dovidio, Victoria L. Brescoll, Mark J. Graham, and Jo Handelsman. 2012. "Science Faculty's Subtle Gender Biases Favor Male Students." Proceedings of the National Academy of Sciences of the United States of America 109(41):1647479. https://doi.org/10.1073/pnas. 1211286109

Neményi, Mária. 2007. "Serdülő Roma Gyerekek Identitás-Stratégiái [Adolescent Identity Strategies for Roma Children]." Educatio (1):84-98.

Nickerson, Raymond S. 1998. "Confirmation Bias: A Ubiquitous Phenomenon in Many Guises." Review of General Psychology 2(2):175-220. https : //doi .org/10.1037/ $1089-2680.2 \cdot 2 \cdot 175$

Niles, Sarah, and Susan Hanson. 2003. "The Geographies of Online Job Search: Preliminary Findings from Worcester, MA." Environment and Planning A 35(7):1223-43. https://doi . org/10.1068/a35253

Pál, Judit, Christoph Stadtfeld, André Grow, and Károly Takács. 2016. "Status Perceptions Matter: Understanding Disliking among Adolescents." Journal of Research on Adolescence 26(4):805-18. https://doi.org/10.1111/jora.12231

Parisi, Domenico, Federico Cecconi, and Francesco Natale. 2003. "Cultural Change in Spatial Environments. The Role of Cultural Assimilation and Internal Changes in Cultures." Journal of Conflict Resolution 47(2):163-79. https : //doi . org/10 .1177/0022002702251025

Pearson, Michael, Christian Steglich, and Tom Snijders. 2006. "Homophily and Assimilation among Sportactive Adolescent Substance Users." Connections 27(1):47-63.

Ponds, Roderik, Frank Van Oort, and Koen Frenken. 2007. "The Geographical and Institutional Proximity of Research Collaboration." Papers in Regional Science 86(3):423-43. https://doi.org/10.1111/j.1435-5957.2007.00126.x

Preciado, Paulina, Tom A. B. Snijders, William J. Burk, Håkan Stattin, and Margaret Kerr. 2012. "Does Proximity Matter? Distance Dependence of Adolescent Friendships." Social Networks 34(1):18-31.

Ridgeway, Cecilia L. 1991. "The Social Construction of Status Value: Gender and Other Nominal Characteristics." Social Forces 70(2):367-86. https://doi.org/10.1093/sf/70. 2.367

Ridgeway, Cecilia L. 2000. "The Formation of Status Beliefs: Improving Status Construction Theory." Pp. 77-102 in Advances in Group Processes, Vol. 17, edited by S. R. Thye and E. J. Lawler. https://doi .org/10.1016/S0882-6145(00)17004-3

Ridgeway, Cecilia L., Kristen Backor, Yan E. Li, Justine E. Tinkler, and Kristan G. Erickson. 2009. "How Easily Does a Social Difference Become a Status Distinction? Gender Matters." American Sociological Review 74(1):44-62. https://doi.org/10.1177/ 000312240907400103 
Ridgeway, Cecilia L., and James W. Balkwell. 1997. "Group Processes and the Diffusion of Status Beliefs." Social Psychology Quarterly 60(1):14-31. https://doi .org/10. 2307/ 2787009

Ridgeway, Cecilia L., Elizabeth H. Boyle, Kathy J. Kuipers, and Dawn T. Robinson. 1998. "How Do Status Beliefs Develop? The Role of Resources and Interactional Experience." American Sociological Review 63(3):331-50. https ://doi .org/10.2307/2657553

Ridgeway, Cecilia L. and Shelley J. Correll. 2006. "Consensus and the Creation of Status Beliefs." Social Forces 85(1):431-53. https ://doi.org/10.1353/sof .2006. 0139

Rouwendal, Jan. 1999. "Spatial Job Search and Commuting Distances." Regional Science and Urban Economics 29(4):491-517. https : //doi .org/10.1016/S0166-0462 (99) 00002-2

Sailer, Kerstin, and Ian McCulloh. 2012. "Social Networks and Spatial Configuration: How Office Layouts Drive Social Interaction." Social Networks 34(1):47-58. https : //doi . org/ $10.1016 / j$.socnet. 2011.05 .005

Sanderson, Stephen K., D. Alex Heckert, and Joshua K. Dubrow. 2005. "Militarist, Marxian, and Non-Marxian Materialist Theories of Gender Inequality: A Cross-Cultural Test." Social Forces 83(4):1425-41. https ://doi .org/10.1353/sof .2005.0080

Schmader, Toni. 2002. "Gender Identification Moderates Stereotype Threat Effects on Women's Math Performance." Journal of Experimental Social Psychology 38(2):194-201. https://doi.org/10.1006/jesp.2001.1500

Stark, Tobias H., and Andreas Flache. 2012. "The Double Edge of Common Interest: Ethnic Segregation as an Unintended Byproduct of Opinion Homophily." Sociology of Education 85(2):179-99. https://doi.org/10.1177/0038040711427314

Steele, Claude M., and Joshua Aronson. 1995. "Stereotype Threat and the Intellectual Test Performance of African Americans." Journal of Personality and Social Psychology 69(5):797811. https://doi.org/10.1037/0022-3514.69.5.797

Stover, Ronald G., and Christine A. Hope. 1984. "Monotheism and Gender Status: A CrossSocietal Study." Social Forces 63(2):335-48. https ://doi .org/10.1093/sf/63.2.335

Tay, Louis, and Ed Diener. 2011. "Needs and Subjective Well-Being Around the World." Journal of Personality and Social Psychology 101(2):354-65. https://doi.org/10.1037/ a0023779

Van Bavel, Jan, and André Grow. 2016. "Introduction: Agent-Based Modelling as a Tool to Advance Evolutionary Population Theory." Pp. 3-27 in Agent-Based Modelling in Population Studies: Concepts, Methods, and Applications, edited by A. Grow and J. Van Bavel. Cham: Springer International Publishing. https://doi.org/10.1093/acprof : oso/9780199608133.003.0001

Wagner, David G., and Joseph Berger. 2002. "Expectation States Theory: An Evolving Research Program." Pp. 41-76 in New Directions in Contemporary Sociological Theory, edited by J. Berger and M. J. Zelditch. Lanham: Rowman \& Littlefield.

Watts, Duncan J. 1999. "Network Dynamics and the Small-World Phenomenon." American Journal of Sociolgy 105(2):493-527. https : //doi .org/10.1086/210318

Watts, Duncan J., and Steven H. Strogatz. 1998. "Collective Dynamics of 'Small-World' Networks." Nature 393(6684):440-42. https : //doi.org/10.1038/30918

Webster Jr., Murray, and Stuart J. Hysom. 1998. "Creating Status Characteristics." American Sociological Review 63(3):351-78. https : //doi .org/10.2307/2657554

Wilensky, Uri. 1999. NetLogo. Evanston, IL: Center for Connected Learning and ComputerBased Modeling, Northwestern University. 
Wong, Ling H., Philippa Pattison, and Garry Robins. 2006. "A Spatial Model for Social Networks." Physica A: Statistical Mechanics and its Applications 360(1):99-120. https: //doi.org/10.1016/j.physa.2005.04.029

World Bank. 2017. "The World Bank Infrastructure Indicators." Retrieved July 19, 2017. http: //data. worldbank. org/topic/infrastructure?view=chart.

Acknowledgements: The first author conducted most of his research for this article as a $\mathrm{PhD}$ student at the Department of Sociology/Interuniversity Centre for Social Science Theory and Methodology of the University of Groningen. We thank the members of the department, in particular the members of the research group Norms and Networks, for their helpful feedback. Earlier versions of this article have been presented at the 2013 Annual Meeting of the American Sociological Association and the 2013 Conference of the International Network of Analytical Sociologists.

André Grow: Centre for Sociological Research, KU Leuven.

E-mail: andre.grow@kuleuven.be

Andreas Flache: Department of Sociology/Interuniversity Centre for Social Science Theory and Methodology, University of Groningen. E-mail: a.flache@rug.nl.

Rafael P. M. Wittek: Department of Sociology/Interuniversity Centre for Social Science Theory and Methodology, University of Groningen. E-mail: r.p.m.wittek@rug.nl. 